\title{
Reversible Partitioning of Nanoparticles at an Oil-Water Interface
}

\author{
Xiaoqing Hua, Michael A. Bevan, * Joelle Frechette * \\ Department of Chemical and Biomolecular Engineering, Johns Hopkins University, Baltimore, \\ Maryland 21218, United States \\ *E-mail: jfrechette@jhu.edu. mabevan@jhu.edu
}

\section{SUPPORTING INFORMATION}

\section{Characterization of Ion-Pair Gold Nanoparticles}

Fourier transform infrared (FTIR) spectroscopy is employed to characterize the ligands on the gold nanoparticles. As described in our previous work, ${ }^{1}$ the presence of $\mathrm{TPeA}^{+}$as part of the ligands on the nanoparticles leads to asymmetric and symmetric $\mathrm{CH}_{3}$ stretching bands at $2955 \pm$ $3 \mathrm{~cm}^{-1}$ and $2870 \pm 2 \mathrm{~cm}^{-1}$, respectively. (Figure S1A) Deprotonation of the carboxylate group is evident from the presence of a band at $1560 \mathrm{~cm}^{-1}$ (asymmetric $\mathrm{COO}^{-}$stretch), and consistent with the presence of ion-pairs between $\mathrm{TPeA}^{+}$ions and deprotonated MHA bound on the gold surface. (Figure S1B) We previously calibrated the $\mathrm{CH}_{2} / \mathrm{CH}_{3}$ infrared absorbance intensity ${ }^{1}$ to quantify the stoichiometry between $\mathrm{TPeA}^{+}$and MHA on the surface of gold nanoparticles. Using the same calibration here we find that the ligands on the particles employed in the experiments have a stoichiometric ratio that is also very close to $1: 1 \mathrm{TPeA}^{\prime} \mathrm{MHA}$. (Table S1)

Gold nanoparticles were imaged using transmission electron microscopy (TEM) (Figure S1C) right after synthesis. Prior to electron microscopy the nanoparticles are dispersed in 2propanol by sonication for $5 \mathrm{~min}$, after which $5 \mu \mathrm{L}$ of the solution is dispensed on carbon-coated copper grids (Electron Microscopy Services, Hatfield, PA). The grid is left to dry at room temperature overnight before imaging, which caused some aggregation of nanoparticles on the grid Ultraviolet-visible spectroscopy is employed to characterize the size and concentration of gold nanoparticles dispersed in $5 \mathrm{mM} \mathrm{TPeA}^{+}$solution at both $\mathrm{pH} 11.0$ and at $\mathrm{pH}$ 11.7. A surface plasma band (SPB) peak at $530 \pm 3 \mathrm{~nm}$ (Figure S1D) indicates the size of gold nanoparticles in aqueous solution around $5 \mathrm{~nm}$ according to our previous characterization ${ }^{1}$. 
Table S1. Measured composition of ion-pair gold nanoparticles as synthesized. Molar ratio of TPeA/MHA was calculated using the calibration for the $\mathrm{CH}_{2} / \mathrm{CH}_{3}$ intensity ratio. ${ }^{1}$

\begin{tabular}{c|c|c} 
& Asym. $\mathrm{CH}_{2} / \mathrm{CH}_{3}$ & Sym. $\mathrm{CH}_{2} / \mathrm{CH}_{3}$ \\
\hline TPeA/MHA molar ratio & $1.0 \pm 0.2$ & $0.9 \pm 0.1$
\end{tabular}
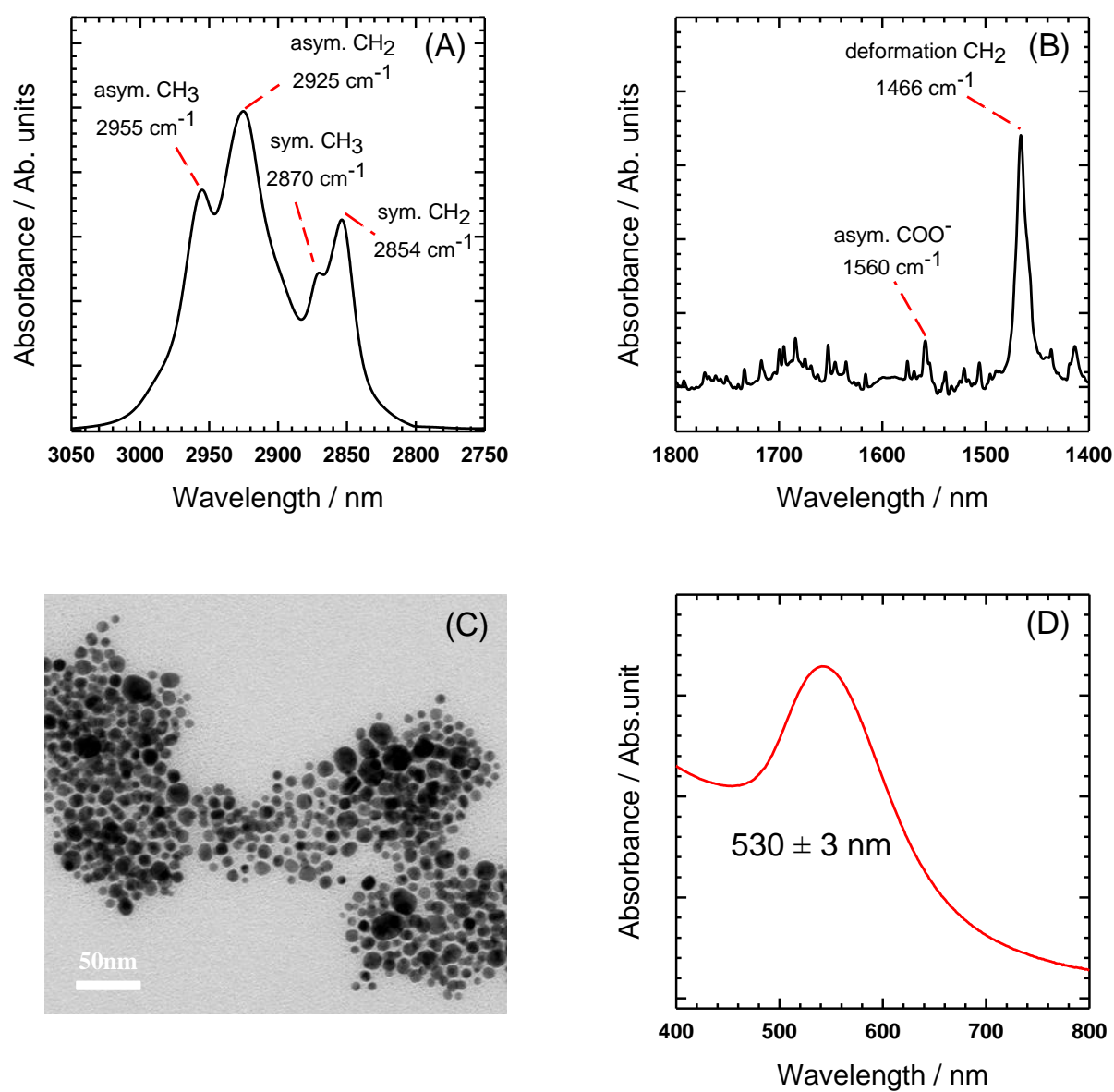

Figure S1. Characterization of gold nanoparticles. (A-B) FTIR characterization of ion-pair gold nanoparticles as synthesized. (C) TEM imaging: gold nanoparticles are dispersed in 2-propanol using sonication and dispersed on TEM grid prior to purification. (D) UV-vis absorption spectrum of NPs dispersed in aqueous solution at $\mathrm{pH} 11.7$.

\section{Effect of purification process on adsorption at oil-water interface}

As described in the main text. NPs are purified prior to experiments by using the interface as a selective filter to remove aggregates and large size particles from synthesis. Figure S2 shows the effect on UV SPB before and after this purification process in reducing NPs polydispersity (left). The removal of aggregates/large particles have a significant effect on adsorption of NPs at oilwater interface, likely due to the preference of aggregates to adsorb compared to single nanoparticles. 

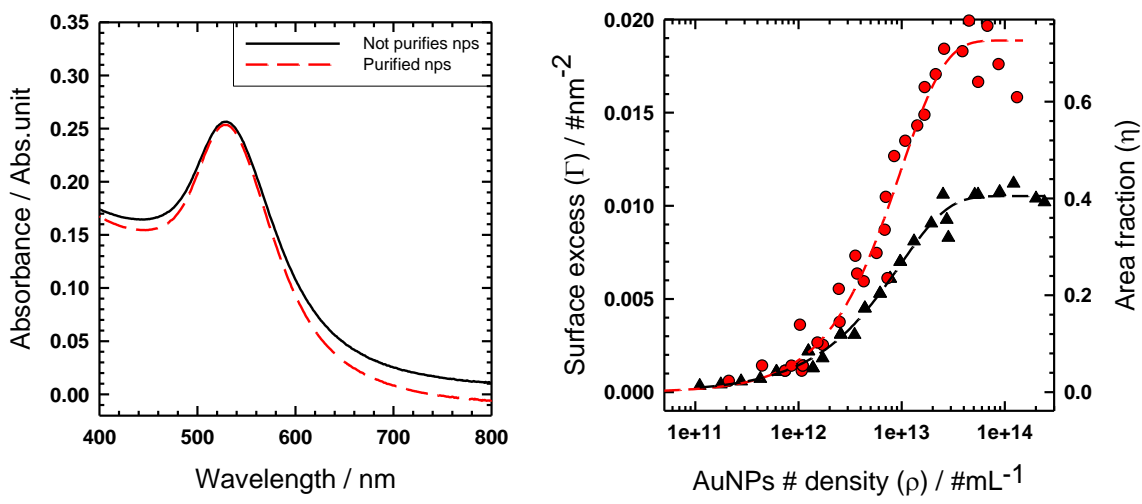

Figure S2 (Left) UV-vis spectra for NPs prior to (black solid line) and after (red dashed line) purification process. (Right) Adsorption prior to (Red cycles) and after (Black triangles) purification process. Dashed lines are to guide the eye.

\section{Adsorption of Gold Nanoparticles at Air-Water Interface}

A key assumption in the determination of the adsorption isotherm is that there is negligible adsorption of nanoparticles at the air-water interface. We verified this assumption by performing control experiments using pendant drop tensiometry. In the control experiments, first the interfacial tension of pure air-water interface is obtained, and a value of $72.8 \pm 0.2 \mathrm{mN} / \mathrm{m}$ (black cycles in Figure S3) is obtained in agreement with a known reported values for a clean air-water interfaces. $^{2}$ Subsequently, we measured the interfacial tension of $5 \mathrm{mM} \mathrm{pH} 11.0$ and $\mathrm{pH} 11.7$ $\mathrm{TPeA}^{+}$aqueous solutions without gold nanoparticles (Figure S3, red hollow cycle representing $\mathrm{pH}$ 11.0, and black hollow triangle standing for $\mathrm{pH} 11.7$ aqueous solution). It is obvious from Figure $\mathrm{S} 3$ that increasing the $\mathrm{pH}$ and adding $\mathrm{TPeA}^{+}$ions to the aqueous solution decreases the surface tension to around $65.4 \pm 0.4 \mathrm{mN} / \mathrm{m}$ (we did not observe a significant effect of $\mathrm{pH}$ on the surface tension based on similar measurements with $\mathrm{KOH}$ solutions). Finally, we repeated the measurements but with the addition of gold nanoparticles at a number density of $3.5 \times 10^{12} / \mathrm{mL}$ in the aqueous phase. As shown in Figure S3, the air-water interfacial tension does not change when particles are present in the aqueous phase (Figure S3, red cycles for $\mathrm{pH} 11.0$ case, and black triangles showing $\mathrm{pH} 11.7$ case), confirming that the gold nanoparticles investigated here

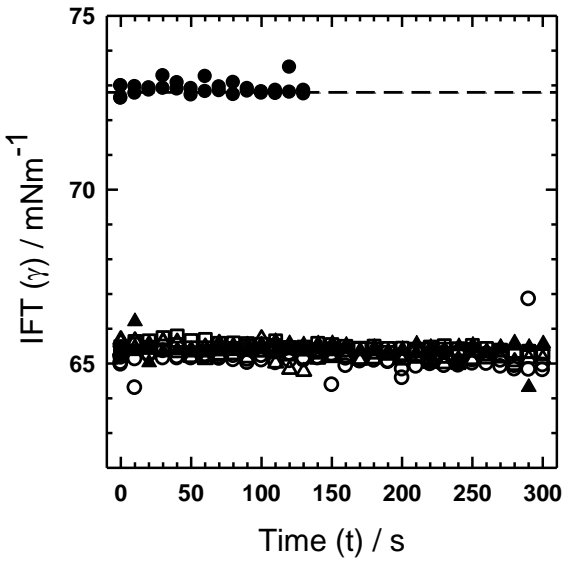
do not adsorb at the air-water interface during the experimental time scale.

Figure S3. Interfacial tension measurements at the air-water interface. (black solid circle) air-water interface, (black hollow circle) air-water interface with $5 \mathrm{mM} \mathrm{pH} 11.0$ aqueous solution, 
(black hollow triangle) air-water interface with $5 \mathrm{mM} \mathrm{pH} 11.7$ aqueous solution, (black hollow square) air-water interface with gold nanoparticles dispersed in $5 \mathrm{mM} \mathrm{pH} 11.0$ aqueous solution, (black solid triangle) air-water interface with gold nanoparticles dispersed in $5 \mathrm{mM} \mathrm{pH} 11.7$ aqueous solution. Black dashed line marking $72.8 \mathrm{mN} / \mathrm{m}$ is used as a reference for pure air-water interface tension.

\section{Estimating Meniscus Area}

The glass vials employed for nanoparticle adsorption have a diameter of $1.3 \mathrm{~cm}$. The curvature of the meniscus formed at the toluene-water interface increases the nominal interfacial area. We imaged the toluene-water interface after equilibration with NPs dispersions (Figure S4A, B). We observe that the contact angle between interface and the cylinder wall is close to $90^{\circ}$, indicating that the interface is approaching a flat surface. The meniscus area was calculated by solving for the height, $h\left(r_{c}\right)$, against the wall, by following eq 1 and integrating for the whole cylindrical wall (which is the glass vial here).

$$
\gamma\left\{\frac{h^{\prime \prime}\left(r_{c}\right)}{\left[1+\left(h^{\prime}\left(r_{c}\right)\right)^{2}\right]^{3 / 2}}+\frac{h^{\prime}\left(r_{c}\right)}{r_{c}\left[1+\left(h^{\prime}\left(r_{c}\right)\right)^{2}\right]^{1 / 2}}\right\}+\Delta \rho g h\left(r_{c}\right)=0
$$

where $\gamma$ is the interfacial tension of the two immiscible solvents, $r_{c}$ is the horizontal distance of interface away from center of cylinder cross-section, $\Delta \rho$ is the density difference between two solvents, $g$ is the gravitational acceleration. The meniscus area can then be solved as a function of contact angle between interface and the cylindrical wall (Figure S4C). Since the imaging indicates a contact angle approaching $90^{\circ}$, and meniscus area is reaching a plateau with contact angle greater than $70^{\circ}$, we use the meniscus area of $1.4 \mathrm{~cm}^{2}$, corresponding to a contact angle of $70^{\circ}$.
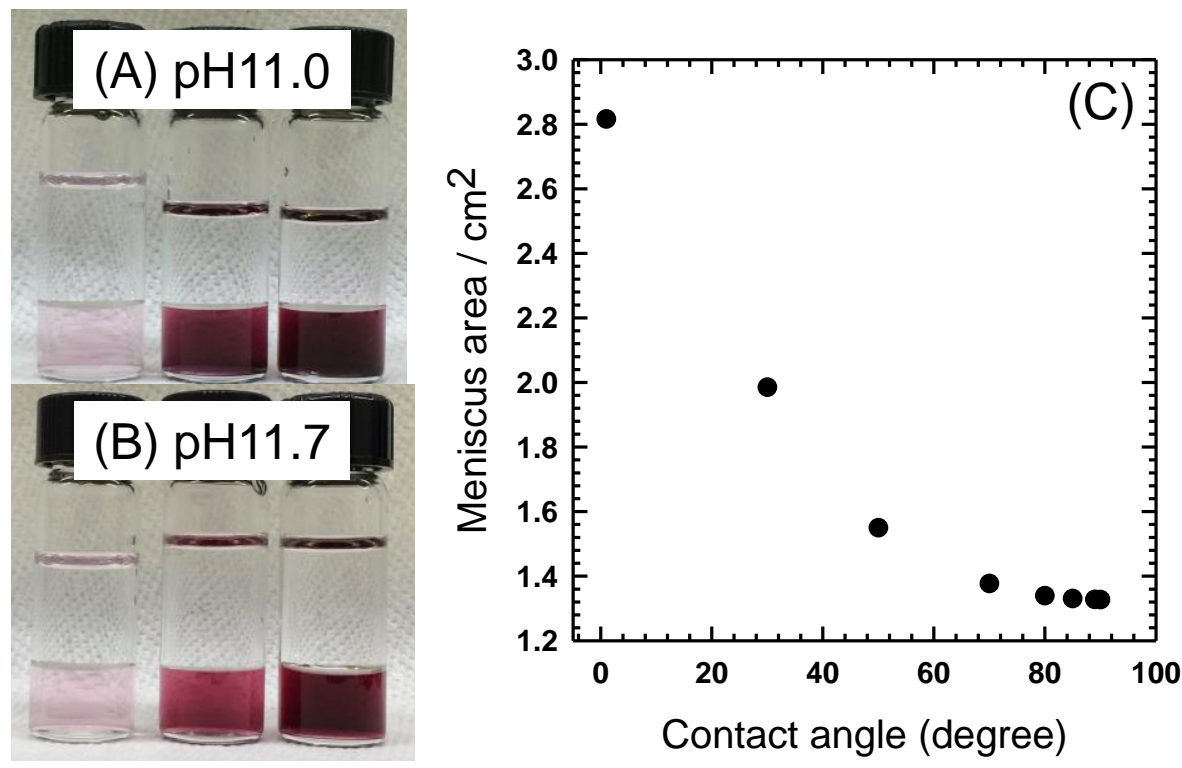

Figure S4. Image of the toluene-water interface at pH 11.0 (A), and $\mathrm{pH} 11.7$ (B). (C) Calculated meniscus area at different contact angles between interface and the wall. 


\section{Effect of TPeA ${ }^{+}$Ions on Interfacial Tension}

It has been noticed that with $\mathrm{TPeA}^{+}$ions only, interfacial tension varies from $35 \mathrm{mN} / \mathrm{m}$ (pure toluene-water interfacial tension) to $23.0 \mathrm{mN} / \mathrm{m}$ (toluene $-5 \mathrm{mM}$ TPeAOH solution interfacial tension). Dynamic interfacial tension was measured in the absence of nanoparticles at the toluene- $5 \mathrm{mM}$ solution interface. The $5 \mathrm{mM}$ solution is a mixture of $5 \mathrm{mM} \mathrm{KOH}(\mathrm{pH} 11.7)$ and 5 $\mathrm{mM}$ TPeAOH ( $\mathrm{pH}$ 11.7) at different ratios, so that a constant $\mathrm{pH}$ and ionic strength can be maintained. It is obvious from Figure S5 that as the concentration of TPeA ions increases, the interfacial tension decreases from $33.6 \mathrm{mN} / \mathrm{m}(5 \mathrm{mM} \mathrm{KOH}$ solution) to $23.0 \mathrm{mN} / \mathrm{m}(5 \mathrm{mM}$ TPeAOH solution).

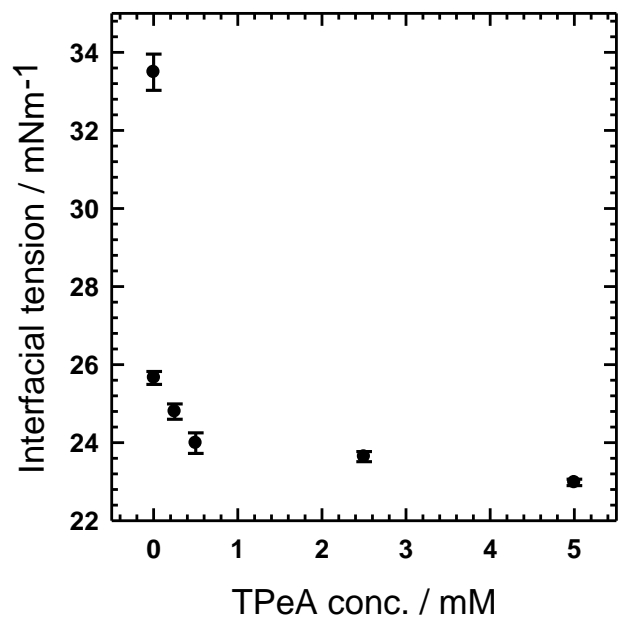

Figure S5 Interfacial tension at toluene- $5 \mathrm{mM}$ pH 11.7 solution interface vs. $\mathrm{TPeA}^{+}$ions concentration in solutions.

Function to Describe the Adsorption Isotherm

A sigmoidal function is used to map the adsorption isotherm data to create the equation of state in Figure 6. We employed the Chapman function (see eq 2).

$$
\Gamma(\rho)=y_{o}+a_{0} \times[1-\exp (-b \times \rho)]^{c}
$$

Table S2. Fitted parameter for the sigmoidal fit used to map the adsorption isotherm in Figure 1.

\begin{tabular}{c|c|c} 
& $\mathrm{pH} 11.0$ & $\mathrm{pH} 11.7$ \\
\hline$y_{o}\left(1 / \mathrm{nm}^{2}\right)$ & $-2.7864 \times 10^{-4}$ & $3.4610 \times 10^{-5}$ \\
\hline$a_{0}\left(1 / \mathrm{nm}^{2}\right)$ & 0.0160 & 0.0105 \\
\hline$b\left(1 / \mathrm{mL}^{2}\right.$ & $8.5945 \times 10^{-14}$ & $9.2449 \times 10^{-14}$
\end{tabular}




$$
\begin{array}{l|l|l}
c \quad 0.5585 & \mid & 0.8257
\end{array}
$$

Fitting Adsorption Isotherm and EOS to Frumkin Equation

The adsorption isotherm at low concentration and the low concentration data in the EOS (first four data points of each $\mathrm{pH}$ ) were fit to the Frumkin equation using MATLAB 2015b by minimizing the distance between each of the data points and the theoretical curve, that is, to minimize the following eq 3 :

$$
d=\frac{1}{m} \sum_{i=1}^{m}\left[X_{i}-\tilde{X}_{i}\right]^{2}
$$

where $X_{i}$ is the experimental data and $\widetilde{X}_{l}$ is the theoretical predicted value. In the adsorption isotherm, $X$ is the bulk NPs density, in EOS, $X$ is the surface pressure $\Pi$. As described by the eqs 5-8 in main text, the fitting parameters for the adsorption isotherm are $a, K, n_{1}$, and $\omega_{0}$, while for the EOS, the fitting parameters are $K, n_{1}$, and $\omega_{0}$.

\section{REFERENCES}

(1). Luo, M.; Olivier, G. K.; Frechette, J. Electrostatic interactions to modulate the reflective assembly of nanoparticles at the oil-water interface. Soft Matter 2012, 8, 11923-11932.

(2). Berg, J. C. An Introduction to Interfaces and Colloids: The Bridge to Nanoscience; World Scientific Publishing Co. Pte. Ltd.2009. 